\title{
REBT WITH PHARMACOTHERAPY IN THE MANAGEMENT OF CHRONIC PAIN- A QUASI- EXPERIMENTAL STUDY
}

\author{
S. Ramesh Kannan', Shanthi Kannan², Kalaiselvi Selvaraj3, Manikandan ${ }^{4}$
}

${ }^{1}$ Associate Professor, Department of Neurology, Pondicherry Institute of Medical Sciences, Pondicherry.

2 ssychologist, Department of Paediatrics, Pondicherry Institute of Medical Sciences, Pondicherry.

${ }^{3}$ Assistant Professor, Department of Community Medicine, Pondicherry Institute of Medical Sciences, Pondicherry.

${ }^{4}$ Assistant Professor cum Statistician, Department of Community Medicine, Pondicherry Institute of Medical Sciences, Pondicherry.

\section{ABSTRACT}

\section{BACKGROUND}

In the recent past, there has been an increase in the number of patients who seek specialist neurological consultations for chronic pain which need longterm pharmacotherapy due to associated biopsychosocial issues.

The aim of the present study was to evaluate the efficacy of Rational Emotive Behaviour Therapy (REBT) and Pharmacotherapy in the management of pain. The objectives of the present study were to determine the associated underlying anxiety, depression and stress in patients with chronic pain; and to compare the efficacy of different treatment modalities like REBT, psychoeducation and pharmacotherapy in the management of chronic pain.

\section{MATERIALS AND METHODS}

150 patients with chronic musculoskeletal pain and primary headache attending the Neurology Outpatient Clinic of a teaching hospital were enrolled through convenience sampling. They were divided into three groups, with 50 patients in each group. Allocation of samples to different groups was done by simple randomisation method. They received the following treatments: (i) Pharmacotherapy with REBT, (ii) Pharmacotherapy with psychoeducation and (iii) regular pharmacotherapy. Numerical pain rating scale (NRS) and Depression, Anxiety and Stress Scale (DASS) were used to assess the patients at entry and at the end of 3 months of treatment to evaluate the severity of pain and psychological comorbidities like depression, anxiety and stress respectively.

Settings and Design- A quasi-experimental intervention study was conducted in a tertiary care hospital in south India. Statistical Analysis- Chi square test, ' $\mathrm{t}$ ' test and Kruskal-Wallis hypothesis test were used to prove the statistical significance.

\section{RESULTS}

A statistically significant, beneficial effect was observed in the Numerical pain rating scale and DASS scores in the group of patients who received pharmacotherapy in combination with Rational Emotive Behaviour Therapy.

\section{CONCLUSION}

We recommend REBT, a simpler form of psychotherapy to be effective in combination with pharmacotherapy in the management of chronic pain.

\section{KEYWORDS}

Chronic Pain, Musculoskeletal Pain, Fibromyalgia, Primary Headache, Rational Emotive Behaviour Therapy, Psychoeducation.

HOW TO CITE THIS ARTICLE: Kannan SR, Kannan S, Selvaraj K, et al. REBT with pharmacotherapy in the management of chronic pain- A quasi-experimental study. J. Evolution Med. Dent. Sci. 2017;6(76):5452-5456, DOI: 10.14260/Jemds/2017/1183

\section{BACKGROUND}

Pain is one of the most common reasons that make the patients to enter the health care setting.

In the recent past, there has been an increase in the number of patients who seek specialist neurological consultations for chronic pain which need longterm pharmacotherapy due to the associated biopsychosocial issues. Suggestions have been made on the relevance of the need for psychological interventions along with medications in the management of pain due to various causes. ${ }^{1}$ Patients with psychological comorbidities like depression, anxiety

Financial or Other, Competing Interest: None.

Submission 07-08-2017, Peer Review 09-09-2017,

Acceptance 15-09-2017, Published 21-09-2017.

Corresponding Author:

Dr. S. Ramesh Kannan,

E-7, PIMS Staff Quarters,

Kanagachettikulam, Kalapet,

Pondicherry-605014.

E-mail: rameshkannanneuro@yahoo.com

DOI: $10.14260 /$ jemds $/ 2017 / 1183$ and stress commonly present with complaints of headache, back pain, neck pain or multiple somatic complaints. ${ }^{2}$ Severity of pain interferes with their quality of life. However, pain relief alone does not improve their ability to function. Extensive research is needed to explain the depth of this association. $^{3}$ The aim of this study was to determine the associated underlying depression, anxiety and stress among patients above the age of 18 who attended the Neurology Outpatient Clinic with chronic musculoskeletal pain and primary headache and to analyse the efficacy of REBT along with pharmacotherapy in the management of chronic pain.

\section{MATERIALS AND METHODS}

We conducted a quasi-experimental intervention study in a tertiary care hospital in South India. A total of 150 patients with primary headache $(n=71)$ and musculoskeletal pain $(\mathrm{n}=79)$ including low back pain, neck pain, and fibromyalgia who came to the Neurology Outpatient Clinic were included in the study through convenience sampling. The diagnosis was ascertained by the Neurologist using standard diagnostic criteria. 4,5 
Patients were excluded from the study if they had chronic pain due to secondary headache, craniofacial pain syndromes like trigeminal neuralgia, entrapment neuropathies, cervical and lumbosacral radiculopathy or systemic diseases producing chronic pain determined by standard investigations which have definitive medical or surgical treatment.

The patients were enrolled by the neurologist before beginning the treatment and reviewed at the end of 3 months. All the patients with headache either had chronic migraine or tension type headache. None of them had trigeminal autonomic cephalalgia. Before initiation and after 3 months of treatment, the pain severity and psychological problems were assessed using Numerical pain rating scale (NRS) and Depression, Anxiety and Stress Scale (DASS) respectively by the psychologist. They were divided into three groups using simple randomisation method.

Group $1(n=50)$ received Pharmacotherapy similar to group 3 and Rational Emotive Behaviour Therapy (REBT). REBT was administered by the psychologist. The therapy involved 8 one-hour sessions. It was held once a week. The treatment protocol of REBT was explained to the patients in the first 2 sessions. In the next four sessions, the patients were explained about the activating events, their irrational beliefs and the consequences in the form of behaviour and emotions. They were requested to introspect on the following: (1) evidence for their beliefs, (2) whether it is consistent with reality, and (3) whether the belief is pragmatic or helpful. They were facilitated to challenge their irrational beliefs and construct effective new beliefs. The last 2 sessions were the final or the 'concluding' sessions, which aimed at preparing members to conduct themselves well when they are confronted with stressful situations in their daily lives. REBT can be completed briefly in as little as five sessions for clearly defined issues. If there were more complex issues, the clients would require longer duration of therapy. ${ }^{6}$

Group 2 were receiving pharmacotherapy similar to group 3 and psychoeducation. Psychoeducation consisted of two 1-hour sessions of providing verbal information, education and guidance regarding the pain; its causes and cognitive distortions which are causative in the emotional disturbances leading to or aggravating the existing pain and providing general guidance in dealing with the present problems. However, it did not include psychotherapeutic techniques like cognitive behavioural therapy. ${ }^{7}$

Patients in group 3 received only pharmacotherapy. They were treated with the following medications. Patients with headache were treated with Propranolol at a dose of $40-80$ mg once daily; musculoskeletal pain comprising of neck pain, back pain and fibromyalgia with Duloxetine at a dose of 30 -
$60 \mathrm{mg}$ at night ${ }^{8,9}$ for a period of 12 weeks. Patients continued to take their regular medications for comorbidities like diabetes mellitus, hypertension and other medical conditions.

Ethics

This study was approved by Institutional ethical committee.

\section{Evaluations}

Patients rated their pain over the previous 24 hours on the NRS $(0=$ no pain and $10=$ worst possible pain). Pain scores were categorised as mild ( 0 to $<4$ ), moderate ( $\geq 4$ to $<7$ ), or severe $(\geq 7$ to $\leq 10) .{ }^{10}$ To measure their psychosocial problems, DASS $21^{11}$ was administered to all the patients and their answers were interpreted by the psychologist.

The DASS is a set of three self-report scales designed to measure the negative emotional states of depression, anxiety and stress. The DASS 21, is available with 7 items per scale. Subjects are asked to use 4-point severity/frequency scales to rate the extent to which they have experienced each state over the past week. Scores for Depression, Anxiety and Stress are calculated by consolidating the scores for the relevant items. The DASS is a reliable questionnaire, free to use and brief to administer in clinical settings; therefore, it is an alternative to the previously used instruments for the screening of anxiety and depression. Furthermore, the subscale stress measures irritability and tension, which are underused in the assessment of patients with chronic pain. ${ }^{12}$

\section{Statistical Analysis}

Difference in baseline characteristics were tested using chi square for sex and diagnosis. Similarly, baseline difference in age was calculated using ' $t$ ' test. Change in score from the baseline was calculated for all the four domains (Depression, Anxiety, Stress and Pain Rating Scale). Since the change in score after treatment did not follow normal distribution and it violated the rule of equal homogeneity of variance, we applied Kruskal-Wallis hypothesis test to prove the statistical significance. Change in score was summarised as median with interquartile range.

\section{RESULTS}

A total of 150 participants were examined. Among 150 participants, 109 (73\%) were female. Majority (34\%) were in the age of 35 - 45 years. 79 (53\%) of the participants had musculoskeletal pain and 71 (47\%) suffered from chronic headache. Groups are comparable based on their baseline characteristics such as age and diagnosis except sex [Table 1]. Comparison of differences in median score shows that improvement in pain was significantly better in group 1 than the other groups by reducing the associated depression, anxiety and stress [Table 2].

\begin{tabular}{|c|c|c|c|c|c|c|c|c|}
\hline \multicolumn{2}{|c|}{ Characteristics } & \multicolumn{2}{|c|}{$\begin{array}{c}\text { Pharmacotherapy } \\
\text { REBT }(\mathrm{N}=50)\end{array}$} & \multicolumn{2}{|c|}{$\begin{array}{c}\text { Pharmacotherapy \& } \\
\text { Psychoeducation }(\mathrm{N}=50)\end{array}$} & \multicolumn{2}{|c|}{$\begin{array}{l}\text { Pharmacotherapy only } \\
\qquad(\mathrm{N}=50)\end{array}$} & \multirow[t]{2}{*}{$\begin{array}{c}P \\
\text { value }\end{array}$} \\
\hline & & Frequency & $\%$ & Frequency & $\%$ & Frequency & $\%$ & \\
\hline \multirow{2}{*}{ Sex } & Male & 12 & 24.0 & 8 & 16.0 & 21 & 42.0 & \multirow{2}{*}{0.02} \\
\hline & Female & 38 & 76.0 & 42 & 84.0 & 29 & 58.0 & \\
\hline \multicolumn{2}{|c|}{ Age in years Mean (SD) } & \multicolumn{2}{|c|}{$43.6(12.1)$} & \multicolumn{2}{|c|}{$41.8(11.3)$} & \multicolumn{2}{|c|}{$39.9(11.9)$} & 0.8 \\
\hline \multirow{2}{*}{ Diagnosis } & Musculoskeletal pain & 29 & 58.0 & 26 & 52.0 & 24 & 48.0 & \multirow[b]{2}{*}{0.3} \\
\hline & Headache & 21 & 42.0 & 24 & 48.0 & 26 & 52.0 & \\
\hline \multicolumn{9}{|c|}{ Table 1. Demographic Profile of the Groups $(N=150)$} \\
\hline
\end{tabular}




\begin{tabular}{|c|c|c|c|c|}
\hline $\begin{array}{c}\text { Factor } \\
\text { Change in Score after Treatment [ } \mathbf{\Delta}] \\
\text { Median (IQR) }\end{array}$ & Group I & Group II & Group III & P value $^{*}$ \\
\hline Depression & $5(3-8)$ & $0(0-1)$ & $0(-1$ to 2$)$ & $<0.001$ \\
\hline Anxiety & $4(3-5)$ & $2(0.8-4)$ & $0(-1$ to 1$)$ & $<0.001$ \\
\hline Stress & $6.5(5-9)$ & $1(0-1)$ & $0(-1$ to 1$)$ & $<0.001$ \\
\hline Pain rating & $6(7-8)$ & $2(1-3.3)$ & $3(1.8-4)$ & $<0.001$ \\
\hline Table 2. Comparison of Mean Difference of Depression, Anxiety, Stress and Pain Rating Scores between Treatment Groups
\end{tabular}

${ }^{*}$ p value by Kruskal-Wallis hypothesis test

\section{DISCUSSION}

The results of this study reiterated the importance of biopsychosocial model of pain management. Increase in psychological factors like anxiety, depression and stress worsened the pain. The findings revealed that Pharmacotherapy I.e. Propranolol used for headache (guideline-recommended preventive therapy) ${ }^{13}$ in combination with REBT (which is a modification of cognitive behavioural therapy), a simpler form of psychotherapy showed significant improvement in reducing the frequency and severity of headache. Various studies have demonstrated the association of pain with psychological factors like social isolation, feeling of threat, insecurity, fatigue and lethargy. ${ }^{14}$

Pain is a perceptual experience created by the brain to warn of threat to specific body regions, along with emotional changes due to memories of previous threatening events. ${ }^{15}$ When pain becomes chronic, sensory input plays a diminished role while affective and cognitive pathways play a more prominent role in the creation of painful perceptions. ${ }^{16}$ Perception of depression, anxiety and stress also share the same pathophysiological pathways as pain which facilitate the central modulation of the pain response, in the periaqueductal grey, amygdala and hypothalamus. These brain areas play a role in depression and anxiety, and when deficits occur in these areas, modulation of signals from the body are disturbed, leading to a more severe experience of pain. Every individual does not respond the same to painful stimuli. Higher levels of depressed mood, anxiety and anger which are common in chronic pain, have been associated with reduced pain thresholds, reduced pain tolerance, and increased patient reported pain. ${ }^{17}$ Furthermore, depression and anxiety induce stress and increases the production of pro-inflammatory cytokines, which may increase pain. ${ }^{18}$

Fibromyalgia (FM) is a prevalent and costly condition worldwide, affecting approximately $2 \%$ of the general population. The diagnosis is based on the history of a typical cluster of symptoms (chronic widespread pain, nonrestorative sleep, physical and/or mental fatigue) that cannot be sufficiently explained by another medical condition. Optimal management should begin with education of patients regarding the current knowledge of FM (including written materials). Management should be a graduated approach with the aim of improving health-related quality of life. The initial focus should ensure active participation of patients in applying healthy lifestyle practices. Aerobic and strengthening exercises should be the foundation of nonpharmacologic management. ${ }^{19}$

Duloxetine is a selective serotonergic, noradrenergic and dopamine reuptake inhibitor (SDNRI) that has been effective in the management of fibromyalgia and other musculoskeletal pain. Duloxetine initiators had less health care utilisation than those started on pregabalin..$^{20}$ None of the patients had significant adverse effects like dizziness, pedal oedema and sedation which were common in pregabalin. Literature review supports duloxetine as well suited for neuropathic pain. ${ }^{21}$

Psychological therapies can be a valuable resource in the treatment of Chronic Widespread Pain (CWP) and fibromyalgia (FM) syndromes that are notoriously difficult to manage with a pharmacological approach. ${ }^{22}$ Passive psychoeducational interventions are relatively easy to implement as they are inexpensive, can be immediately applied and readily incorporated for anxiety and depression. ${ }^{23}$ Depressive symptoms, as measured by the DASS, were associated more strongly with cognitive variables like irrational beliefs than pain in our sample of patients. ${ }^{24}$ REBT targets unhelpful beliefs which lead to pain. Rational Emotive Behaviour Therapy distinguishes itself from other cognitive-behavioural approaches by placing irrational and rational beliefs at its core. Flexible, logical beliefs which are consistent with reality are considered to be rational and rigid beliefs which are inconsistent with reality are considered as irrational beliefs in REBT.

'ABC framework' considers the role of cognitive appraisal in the generation of emotion and it also facilitates therapy, as it is easy for clients to understand the antecedents to their emotions and behaviours. Most prominently, it enables clients to realise that it is not outside events (A) that cause their dysfunctional reactions (C); rather it is their irrational beliefs (B), and thus, they are in control of how they respond to adversity because they can have autonomy over their beliefs. ${ }^{25}$

The central aim of REBT is to reduce irrational beliefs, encouraging healthy negative emotions (HNEs). This is done using a systematic disputation (D) process, which entails the practitioner helping the client to challenge specific irrational beliefs following which an effective new belief is constructed (E), ${ }^{26}$ hence REBT is remembered as 'ABCDE'.

Unhealthy associates of irrational beliefs such as anger, guilt and shame, and psychopathological conditions including depression, anxiety, and suicidal thoughts were addressed during the REBT sessions. The goal of REBT is to enhance and maintain emotional and behavioural functionality, which helps to drive longterm goal achievement. It is important to recognise that REBT is also a preventive approach that can bolster rational beliefs and mental health, and is not just about providing a solution to irrational beliefs and mental illhealth. Irrational beliefs like needing to excel in all endeavours, feeling terrible when things don't happen as expected, worries of future misfortunes and impossibility to overcome the effects of past history, were the causes of depression. Irrational beliefs have been shown to affect physical health leading to a higher frequency of chronic illnesses. There are clinical and non-clinical mental health 
outcomes. REBT offers enduring change in 1 to 20 sessions of cognitive therapies with approximately 50 minutes weekly individual sessions. 27

This study has the following strengths. This is the first study of its kind analysing the effect of REBT in chronic headache and musculoskeletal pain. Since these conditions usually have psychosocial determinants and influencers which may have a role in worsening the degree of pain, the results of the present study will form the basis for future studies on patients with the abovementioned conditions.

\section{Limitations}

This study focused on the assessment of emotions and pain rating. Further studies are required to measure changes in cognition, beliefs and behaviour from a pain perspective. These effects are promising, but considerable uncertainty remains around the estimates of effect of REBT on individual conditions of pain as these results come from a small number of pain sufferers. These therapies should be explored in larger population. Future studies are needed using larger groups of patients in other chronic neurological disorders with poor response to current pharmacotherapy.

\section{Implications in the Clinical Practice}

These modalities can be tried in patients with neurogenic pain which shows poor response even after adequate doses of medications; and also, to lower the doses of medications without much adverse reactions.

\section{CONCLUSION}

Pharmacotherapy when combined with psychotherapeutic procedures like REBT is found to be effective in the treatment of patients presenting to the neurologist with chronic pain by reducing the associated psychological comorbidity like depression, anxiety and stress. This study reiterates the importance of a psychologist in the Neurology team of any tertiary care institution.

\section{REFERENCES}

[1] Thieme K, Mathys M, Turk DC. Evidenced-Based guidelines on the treatment of fibromyalgia patients: are they consistent and if not, why not? have effective psychological treatments been overlooked? J Pain 2017;18(7):747-56.

[2] Janwantanakul P, Sitthipornvorakul E, Paksaichol A. Risk factors for the onset of nonspecific low back pain in office workers: a systematic review of prospective cohort studies. J Manipulative Physiol Ther 2012;35(7):568-77.

[3] Lucchetti G, Oliveira AB, Mercante JP, et al. Anxiety and fear-avoidance in musculoskeletal pain. Curr Pain Headache Rep 2012;16(5):399-406.

[4] Fatemi G, Fang MA, Breuer P, et al. Deconstructing chronic low back pain in the older adult - step by step evidence and expert-based recommendations for evaluation and treatment part III: Fibromyalgia syndrome. Pain Med 2015;16(9):1709-19.

[5] Lebedeva ER, Gurary NM, Gilev DV, et al. Prospective testing of ICHD-3 beta diagnostic criteria for migraine with aura and migraine with typical aura in patients with transient ischemic attacks. Cephalalgia 2017:333102417702121.

[6] Digiuseppe RA, Doyle CA, Dryden W, et al. A Practitioner's guide to rational emotive behavior therapy. $3^{\text {rd }}$ edn. New York: Oxford University Press 2014.

[7] Donke T, Griffiths KM, Cuijpers P, et al. Psychoeducation for depression, anxiety and psychological distress: a meta-analysis. BMC Med 2009;7:79.

[8] Murakami M, Osada K, Mizuno H, et al. A randomized, double-blind, placebo-controlled phase III trial of duloxetine in Japanese fibromyalgia patients. Arthritis Res Ther 2015;17:224.

[9] Kim SC, Landon JE, Lee YC. Patterns of health care utilization related to initiation of amitriptyline, duloxetine, gabapentin, or pregabalin in fibromyalgia. Arthritis Res Ther 2015;17:18.

[10] Hierrmstad MJ, Fayers PM, Haugen DF, et al. Studies comparing numerical rating scales, verbal rating scales, and visual analogue scales for assessment of pain intensity in adults: a systematic literature review. JPSM 2011;41(6):1073-93.

[11] Lovibond SH, Lovibond PF. Manual for the depression anxiety stress scales. $2^{\text {nd }}$ edn. Sydney, N.S.W: Psychology Foundation of Australia c1995.

[12] Mafi JN, Edwards ST, Pedersen NP, et al. Trends in the ambulatory management of headache: analysis of NAMCS and NHAMCS data 1999-2010. J Gen Intern Med 2015;30(5):548-55.

[13] Wiech K. Deconstructing the sensation of pain: the influence of cognitive processes on pain perception. Science 2016;354(6312):584-7.

[14] Tracey I, Mantyh PW. The cerebral signature for pain perception and its modulation. Neuron 2007;55(3):377-91.

[15] Ossipov MH, Dussor GO, Porreca F. Central modulation of pain. J Clin Invest 2010;120(11):377987.

[16] Van Middendorp H, Lumley MA, Jacobs JW, et al. The effects of anger and sadness on clinical pain reports and experimentally-induced pain thresholds in women with and without fibromyalgia. Arthritis Care Res (Hoboken) 2010;62(10):1370-6.

[17] Felger JC, Lotrich FE. Inflammatory cytokines in depression: neurobiological mechanisms and therapeutic implications. Neuroscience 2013;246:199-229.

[18] Goldenberg DL. Multidisciplinary modalities in the treatment of fibromyalgia. J Clin Psychiatry 2008;69(Suppl 2):30-4.

[19] Smith HS, Smith EJ, Smith BR. Duloxetine in the management of chronic musculoskeletal pain. Ther Clin Risk Manag 2012;8:267-77.

[20] McBeth J, Prescott G, Scotland G, et al. Cognitive behavior therapy, exercise or both for treating chronic widespread pain. Arch Intern Med 2012;172(1):4857.

[21] Cuijpers P. Bibliotherapy in unipolar depression: a meta-analysis. J Behav Ther Exp Psychiatry 1997;28(2):139-47. 
[22] Nicholas MK, Coulston CM, Asghari A, et al. Depressive symptoms in patients with chronic pain. Med J Aust 2009;190(Suppl 7):S66-70.

[23] David D, Szentagotai A, Lupu V, et al. Rational emotive behavior therapy, cognitive therapy and medication in the treatment of major depressive disorder: a randomized clinical trial, posttreatment outcomes and six-month follow-up. J Clin Psychol 2008;64(6):72846.

[24] David D, Schnur J, Belloiu A. Another search for the "hot" cognitions: appraisal, irrational beliefs, attributions and their relation to emotion. J Ration Emot Cogn Behav Ther 2002;20(2):93-131.
[25] David D, Szentagotai A, Eva K, et al. A synopsis of rational-emotive behavior therapy (REBT): fundamental and applied research. J Ration Emot Cogn Behav Ther 2005;23(3):175-221.

[26] Browne CM, Dowd ET, Freeman A. Rational and irrational beliefs and psychopathology. In: David D, Lynn SJ, Ellis A. eds. Rational and irrational beliefs in human functioning and disturbances: implications for research, theory and practice. New York, NY: Oxford University Press 2010.

[27] Mahigir F, Khanehkeshi A, Karimi A. Psychological treatment for pain among cancer patients by rationalemotive behavior therapy-efficacy in both India and Iran. Asian Pac J Cancer Prev 2012;13(9):4561-5. 